\title{
WEIERSTRASS STRUCTURE AND EIGENVALUE PLACEMENT OF REGULAR MATRIX PENCILS UNDER LOW RANK PERTURBATIONS*
}

\author{
ITZIAR BARAGAÑA ${ }^{\dagger}$ AND ALICIA ROCA ${ }^{\ddagger}$
}

\begin{abstract}
We solve the problem of determining the Weierstrass structure of a regular matrix pencil obtained by a low rank perturbation of another regular matrix pencil. We apply the result to find necessary and sufficient conditions for the existence of a low rank perturbation such that the perturbed pencil has prescribed eigenvalues and algebraic multiplicities. The results hold over fields with sufficient number of elements.
\end{abstract}

Key words. regular matrix pencil, Weierstrass structure, low rank perturbation, matrix spectral perturbation theory

AMS subject classifications. 15A22, 47A55, 15A18

DOI. $10.1137 / 18 \mathrm{M} 1200245$

1. Introduction. In the last few decades the problem of low rank perturbation has been widely studied by different authors from different points of view. Given a matrix $A$, the problem consists of characterizing the invariants of $A+P$ with respect to a given equivalence relation, where $P$ is a matrix of bounded rank. As pointed out in [6], the problem is equivalent to the rank distance problem, i.e., the problem of finding two matrices with prescribed invariants, such that their difference has bounded rank.

Different requirements on the matrices $A, P$ and a matrix $B$ equivalent to $A+P$, on their domain and on the equivalence relation, lead to different types of problems. Additionally, in many contributions in the area the problem is addressed generically, i.e., the perturbation $P$ belongs to an open and dense subset of the set of matrices with rank less than or equal to $r$, for a given integer $r$ (see, for instance, $[1,2,3,4,5$, $14,15,17,18]$ and the references therein). In other cases, the matrix $P$ is an arbitrary perturbation belonging to the whole set of matrices of rank less than or equal to $r$. In this paper we follow the second approach. Some results related to the problem from this point of view can be found in $[6,9,11,13,19,20,21,22]$. We next analyze most of them.

For matrices over a principal ideal domain the problem of characterizing the invariant factors of $A+P$, where $P$ is a matrix with $\operatorname{rank}(P) \leq r$, has been solved in [21] for $r=1$ and in [13] for the general case (see Lemma 4.1). For square matrices over a field and the similarity relation, a solution is given in [20] and [22] (see Proposition 4.9). For $r=1$ the problem was already solved in [21]. The case where the perturbation $P$ has fixed $\operatorname{rank}(\operatorname{rank}(P)=r)$ has been solved in [20] for square

\footnotetext{
${ }^{*}$ Received by the editors July 13, 2018; accepted for publication (in revised form) by F. M. Dopico January 25, 2019; published electronically DATE.

http://www.siam.org/journals/simax/x-x/M120024.html

Funding: The work of the first and second authors was partially supported by MINECO grants MTM2017-83624-P and MTM2017-90682-REDT. The work of the first author was also partially supported by UPV/EHU grant GIU16/42.

${ }^{\dagger}$ Departamento de Ciencia de la Computación e I.A., Facultad de Informática, Universidad del País Vasco, UPV/EHU, Apartado 649, 20080 Donostia-San Sebastián, Spain (itziar. baragana@ehu.eus).

${ }^{\ddagger}$ Departamento de Matemática Aplicada, IMM, Universitat Politècnica de València, 46022 Valencia, Spain (aroca@mat.upv.es).
} 
matrices over algebraically closed fields, and for matrices over principal ideal domains in [19]. More recently, in [6], the low rank perturbation problem is solved for pairs of matrices and the feedback equivalence relation.

It is well known that two matrices $A, B \in \mathbb{F}^{n \times n}, \mathbb{F}$ a field, are similar if and only if their characteristic matrices $s I_{n}-A$ and $s I_{n}-B$ are equivalent as polynomial matrices (see, for instance, [7, Chap. 2], [8, Chap. 6]). Therefore, the results in $[20,21,22]$ hold for regular pencils having only finite invariant factors; hence they give a solution to the low rank perturbation problem for this type of pencils and for a constant perturbation. In the same way, given a matrix pair $\left(A_{1}, A_{2}\right)$ with $A_{1} \in \mathbb{F}^{n \times n}$, $A_{2} \in \mathbb{F}^{n \times m}$, we can associate to $\left(A_{1}, A_{2}\right)$ the singular matrix pencil $\left[\begin{array}{ll}s I_{n}-A_{1} & A_{2}\end{array}\right]$. Two matrix pairs are feedback equivalent if and only if the associated pencils are strictly equivalent (see, for instance, [10, Chap. IX]). Then, the result in [6] holds for singular matrix pencils of the form $\left[s I_{n}-A_{1} A_{2}\right]$ and the strict equivalence relation of matrix pencils, when the perturbation matrix is constant.

In this paper we solve the low rank perturbation problem for regular matrix pencils and the strict equivalence relation, when the perturbation matrix is allowed to be a pencil (pencil perturbation problem). The general pencil perturbation problem for arbitrary singular pencils remains open.

A complete system of invariants for the strict equivalence of regular matrix pencils is formed by the invariant factors (equivalently, the finite elementary divisors) and the infinite elementary divisors. We refer to them as the Weierstrass structure of the pencil. Given a regular matrix pencil $A(s)$, we obtain necessary conditions for the Weierstrass structure of the pencil $A(s)+P(s)$, when $P(s)$ is a pencil with $\operatorname{rank}(P(s)) \leq r$ and $A(s)+P(s)$ is regular, which hold for arbitrary fields. Conversely, we prove that the necessary conditions obtained are sufficient, but in this case we need to impose a condition on the field: we need the field to have a sufficient number of elements (see Remark 4.15).

As mentioned above, the solutions to the perturbation problem provided in [20, $21,22]$ are also solutions to our problem in the case where the pencils do not have infinite elementary divisors. Notice that the perturbation matrix $\mathrm{P}$ is required there to be constant. In the more general statement of perturbation of polynomial matrices of $[13,19,21]$, the only invariants involved in the equivalence relation are the (finite) invariant factors, and the perturbation matrix can be a polynomial matrix of any degree. In this paper, the perturbation problem is solved for arbitrary regular pencils, therefore including the case where they have infinite elementary divisors, and the perturbation matrix is allowed to be a polynomial matrix of degree at most one.

Moreover, the result we obtain allows us to generalize the solution given in [9] to the eigenvalue placement problem. Namely, given a regular matrix pencil $A(s)$, we obtain necessary and sufficient conditions for the existence of a pencil $P(s)$ with $\operatorname{rank}(P(s)) \leq r$ such that $A(s)+P(s)$ is regular with prescribed eigenvalues and algebraic multiplicities. In [9] the solution to this problem is obtained for $r=1$ and for complex or real pencils.

For complex regular matrix pencils, generic low rank perturbations have been studied, for instance, in $[4,5]$. There, an eigenvalue of the pencil $A(s)$ is fixed and the spectral behavior of the eigenvalue after perturbation is generically characterized. In our result all of the possible achievable partial multiplicities of all of the eigenvalues are characterized, therefore including those of the generic behavior. The same type of problem for singular matrix pencils was studied in [3] and for structured regular pencils, for example, in $[1,2]$. In both cases, the problem is addressed generically.

The paper is organized as follows. In section 2 we introduce the notation, basic 
definitions, and preliminary results. In section 3 the problems to be studied in the paper are established. In section 4 we provide in Theorem 4.13 a solution to the stated low rank perturbation problem. Section 5 is devoted to solving the eigenvalue placement problem, and the solution is given in Theorem 5.4. Finally, in section 6 we summarize the main contributions of the paper.

2. Preliminaries. Let $\mathbb{F}$ be a field. $\mathbb{F}[s]$ denotes the ring of polynomials in the indeterminate $s$ with coefficients in $\mathbb{F}$, and $\mathbb{F}[s, t]$ denotes the ring of polynomials in two variables $s, t$ with coefficients in $\mathbb{F}$. We denote by $\mathbb{F}^{m \times n}, \mathbb{F}[s]^{m \times n}$, and $\mathbb{F}[s, t]^{m \times n}$ the vector spaces of $m \times n$ matrices with elements in $\mathbb{F}, \mathbb{F}[s]$, and $\mathbb{F}[s, t]$, respectively. $\mathrm{Gl}_{n}(\mathbb{F})$ will be the general linear group of invertible matrices in $\mathbb{F}^{n \times n}$. A matrix $U(s) \in \mathbb{F}[s]^{n \times n}$ is unimodular if $0 \neq \operatorname{det}(U(s)) \in \mathbb{F}$, i.e., it is a unit in the ring $\mathbb{F}[s]^{n \times n}$.

Given a polynomial matrix $G(s) \in \mathbb{F}[s]^{m \times n}$, the degree of $G(s)$, denoted by $\operatorname{deg}(G(s))$, is the maximum of the degrees of its entries. The normal rank of $G(s)$, denoted by $\operatorname{rank}(G(s))$, is the order of the largest nonidentically zero minor of $G(s)$, i.e., it is the rank of $G(s)$ considered as a matrix on the field of fractions of $\mathbb{F}[s]$. The determinantal divisor of order $k$ of $G(s)$, denoted by $D_{k}(s)$, is the monic greatest common divisor of the minors of order $k$ of $G(s), 1 \leq k \leq \operatorname{rank}(G(s))$.

Two polynomial matrices $G(s), H(s) \in \mathbb{F}[s]^{m \times n}$ are equivalent to $(G(s) \sim H(s))$ if there exist unimodular matrices $U(s) \in \mathbb{F}[s]^{m \times m}, V(s) \in \mathbb{F}[s]^{n \times n}$ such that $G(s)=$ $U(s) H(s) V(s)$. If $G(s) \in \mathbb{F}[s]^{m \times n}$ and $\operatorname{rank}(G(s))=\rho$, it is well known (see, for example, [8, Chap. 6]) that $G(s)$ is equivalent to a unique matrix of the form

$$
S(s)=\left[\begin{array}{cc}
\operatorname{diag}\left(\gamma_{1}(s), \ldots, \gamma_{\rho}(s)\right) & 0 \\
0 & 0
\end{array}\right]
$$

where $\gamma_{1}(s), \ldots, \gamma_{\rho}(s)$ are monic polynomials and $\gamma_{1}(s)|\cdots| \gamma_{\rho}(s)$. Moreover,

$$
D_{k}(s)=\gamma_{1}(s), \ldots, \gamma_{k}(s), \quad 1 \leq k \leq \rho,
$$

which means that

$$
\gamma_{k}(s)=\frac{D_{k}(s)}{D_{k-1}(s)}, \quad 1 \leq k \leq \rho \quad\left(D_{0}(s)=1\right)
$$

The matrix $S(s)$ is the Smith form of $G(s)$ and the polynomials $\gamma_{1}(s), \ldots, \gamma_{\rho}(s)$ are the invariant factors of $G(s)$. We will take $\gamma_{i}(s):=1$ for $i<1$ and $\gamma_{i}(s):=0$ for $i>\rho$.

The invariant factors form a complete system of invariants for the equivalence of polynomial matrices, i.e., two polynomial matrices $G(s), H(s) \in \mathbb{F}[s]^{m \times n}$ are equivalent if and only if they have the same invariant factors.

A matrix pencil is a polynomial matrix $G(s) \in \mathbb{F}[s]^{m \times n} \operatorname{such}$ that $\operatorname{deg}(G(s)) \leq 1$. The pencil is regular if $m=n$ and $\operatorname{det}(G(s))$ is not the zero polynomial. Otherwise, it is singular.

Two matrix pencils $G(s)=G_{0}+s G_{1}, H(s)=H_{0}+s H_{1} \in \mathbb{F}[s]^{m \times n}$ are strictly equivalent $(G(s) \stackrel{\text { s.e. }}{\sim} H(s))$ if there exist invertible matrices $Q \in \mathrm{Gl}_{m}(\mathbb{F}), R \in \mathrm{Gl}_{n}(\mathbb{F})$ such that $G(s)=Q H(s) R$. Equivalently, $G_{0}=Q H_{0} R, G_{1}=Q H_{1} R$.

It is immediate that if $G(s) \stackrel{\text { s.e. }}{\sim} H(s)$, then $G(s) \sim H(s)$. Moreover, if $n=m$, $\operatorname{det}\left(G_{1}\right) \neq 0$ and $\operatorname{det}\left(H_{1}\right) \neq 0$, then $G(s) \stackrel{\text { s.e. }}{\sim} H(s)$ if and only if $G(s) \sim H(s)$ (see, for instance, $[8$, Chap. 12, Theorem 1]). 
If $\gamma_{1}(s)|\cdots| \gamma_{\rho}(s)$ are the invariant factors of $G(s)=G_{0}+s G_{1} \in \mathbb{F}[s]^{m \times n}$, then the invariant factors of the matrix pencil $\bar{G}(t)=t G_{0}+G_{1} \in \mathbb{F}[t]^{m \times n}$ can be expressed as

$$
\bar{\gamma}_{i}(t)=k_{i} t^{q_{i}} t^{\operatorname{deg}\left(\gamma_{i}\right)} \gamma_{i}\left(\frac{1}{t}\right), \quad 1 \leq i \leq \rho,
$$

for some integers $q_{i} \geq 0$, where $0 \neq k_{i} \in \mathbb{F}$ are scalars such that $\bar{\gamma}_{i}(t)$ are monic. If $q_{i}>0$, then $t^{q_{i}}$ is an infinite elementary divisor of $G(s)$. The infinite elementary divisors of $G(s)$ exist if and only if $\operatorname{rank}\left(G_{1}\right)<\operatorname{rank}(G(s))$.

The exponents $q_{i}$ are the partial multiplicities at infinity of $G(s)$ and we will denote them by $m_{i}(\infty, G(s)):=q_{i}$. Then, $m_{1}(\infty, G(s)) \leq \cdots \leq m_{\rho}(\infty, G(s))$.

Given $G(s)=G_{0}+s G_{1} \in \mathbb{F}[s]^{m \times n}$, with $\operatorname{rank}(G(s))=\rho$, the homogeneous pencil associated to $G(s)$ is

$$
G(s, t)=t G_{0}+s G_{1} \in \mathbb{F}[s, t]^{m \times n}
$$

and the homogeneous determinantal divisor of order $k$ of $G(s)$, denoted by $\Delta_{k}(s, t)$, is the greatest common divisor of the minors of order $k$ of $G(s, t), 1 \leq k \leq \rho$. We will assume that $\Delta_{k}(s, t)$ is monic with respect to $s$. The homogeneous determinantal divisors of $G(s)$ are homogeneous polynomials and $\Delta_{k-1}(s, t) \mid \Delta_{k}(s, t), 1 \leq k \leq \rho$. Defining

$$
\Gamma_{k}(s, t)=\frac{\Delta_{k}(s, t)}{\Delta_{k-1}(s, t)}, \quad 1 \leq k \leq \rho \quad\left(\Delta_{0}(s, t)=1\right),
$$

if $\gamma_{1}(s)|\cdots| \gamma_{\rho}(s)$ and $\bar{\gamma}_{1}(t)|\cdots| \bar{\gamma}_{\rho}(t)$ are the invariant factors of $G(s)$ and $\bar{G}(t)=t G_{0}+G_{1}$, respectively, then

$$
\gamma_{i}(s)=\Gamma_{i}(s, 1), \quad \bar{\gamma}_{i}(t)=k_{i} \Gamma_{i}(1, t), \quad 1 \leq i \leq \rho \quad\left(0 \neq k_{i} \in \mathbb{F}\right),
$$

and

$$
\Gamma_{i}(s, t)=t^{m_{i}(\infty, G(s))} t^{\operatorname{deg}\left(\gamma_{i}\right)} \gamma_{i}\left(\frac{s}{t}\right), \quad 1 \leq i \leq \rho .
$$

As a consequence, $\Gamma_{1}(s, t)|\cdots| \Gamma_{\rho}(s, t)$. The polynomials $\Gamma_{1}(s, t), \ldots, \Gamma_{\rho}(s, t)$ are called the homogeneous invariant factors of $G(s)$. For details, see [7, Chap. 2], [8, Chap. 12]. We will take $\Gamma_{i}(s, t):=1$ for $i<1$ and $\Gamma_{i}(s, t):=0$ for $i>\rho$. Observe that the homogeneous invariant factors of $\bar{G}(t)=t G_{0}+G_{1}$ are $\bar{\Gamma}_{i}(t, s)=k_{i} \Gamma_{i}(s, t), 0 \neq$ $k_{i} \in \mathbb{F}$.

The homogeneous invariant factors form a complete system of invariants for the strict equivalence of regular pencils. A proof of the following theorem can be found, for instance, in [8, Chap. 12] for infinite fields and in [16, Chap. 2] for arbitrary fields.

THEOREM 2.1 (Weierstrass). Two regular matrix pencils are strictly equivalent if and only if they have the same homogeneous invariant factors.

Notice that the invariant factors and the infinite elementary divisors determine the homogeneous invariant factors. As a consequence, two regular matrix pencils are strictly equivalent if and only if they have the same invariant factors and the same infinite elementary divisors.

We denote by $\overline{\mathbb{F}}$ the algebraic clousure of $\mathbb{F}$. The finite spectrum of a regular pencil $G(s)=G_{0}+s G_{1} \in \mathbb{F}[s]^{n \times n}$ is defined as

$$
\Lambda_{f}(G(s))=\{\lambda \in \overline{\mathbb{F}}: \operatorname{det}(G(\lambda))=0\} .
$$

If $\gamma_{1}(s)|\cdots| \gamma_{n}(s)$ are the invariant factors of $G(s)$, then we can write

$$
\gamma_{i}(s)=\prod_{\lambda \in \Lambda_{f}(G(s))}(s-\lambda)^{m_{i}(\lambda, G(s))},
$$


where $0 \leq m_{1}(\lambda, G(s)) \leq \cdots \leq m_{n}(\lambda, G(s))$ are called the partial multiplicities at $\lambda$ of $G(s)$. The spectrum of $G(s)$ is $\Lambda(G(s))=\Lambda_{f}(G(s))$ if $\operatorname{det}\left(G_{1}\right) \neq 0$ and $\Lambda(G(s))=$ $\Lambda_{f}(G(s)) \cup\{\infty\}$ if $\operatorname{det}\left(G_{1}\right)=0$. The elements $\lambda \in \Lambda(G(s))$ are the eigenvalues of $G(s)$.

If $\lambda \in \overline{\mathbb{F}} \backslash \Lambda(G(s))$, we put $m_{1}(\lambda, G(s))=\cdots=m_{n}(\lambda, G(s))=0$. For $\lambda \in \overline{\mathbb{F}} \cup\{\infty\}$, we will agree that $m_{i}(\lambda, G(s))=0$ for $i<1$ and $m_{i}(\lambda, G(s))=\infty$ for $i>n$. We denote by $\left(w_{1}(\lambda, G(s)), \ldots, w_{n}(\lambda, G(s))\right)$ the conjugate partition of the partition $\left(m_{n}(\lambda, G(s)), \ldots, m_{1}(\lambda, G(s))\right)$, i.e.,

$$
w_{i}(\lambda, G(s))=\#\left\{j \in\{1, \ldots, n\}: m_{j}(\lambda, G(s)) \geq i\right\}, \quad 1 \leq i \leq n .
$$

The algebraic multiplicity of $\lambda$ in $G(s)$ is $\mu_{a}(\lambda, G(s)):=\sum_{i=1}^{n} m_{i}(\lambda, G(s))$, and the geometric multiplicity is $\mu_{g}(\lambda, G(s)):=\#\left\{i \in\{1, \ldots, n\}: m_{i}(\lambda, G(s))>0\right\}$, i.e., $\mu_{g}(\lambda, G(s))=w_{1}(\lambda, G(s))$.

The following technical result characterizes some inequality relations between the elements of two partitions of nonnegative integers and those of their conjugate partitions.

LeMma 2.2 (see [12, Lemma 3.2]). Let $p_{1} \geq p_{2} \geq \cdots \geq 0$ and $p_{1}^{\prime} \geq p_{2}^{\prime} \geq \cdots \geq 0$ be partitions of $n$ and $n^{\prime}$ with conjugate partitions $q_{1} \geq q_{2} \geq \cdots \geq 0$ and $q_{1}^{\prime} \geq q_{2}^{\prime} \geq$ $\cdots \geq 0$. Let $r \in \mathbb{N}$. Then $q_{i}^{\prime} \geq q_{i+r}$ and $q_{i} \geq q_{i+r}^{\prime}$ for all $i>0$ if and only if $\left|p_{i}-p_{i}^{\prime}\right| \leq r$ for all $i>0$.

In the next lemma, whose proof is straightforward, we show that conditions of divisibility between homogeneous invariant factors can be expressed in terms of divisibility between invariant factors and infinite elementary divisors.

Lemma 2.3. Let $\gamma(s), \omega(s) \in \mathbb{F}[s]$ be monic polynomials, and let $m, m^{\prime}$ be nonnegative integers. If $\Gamma(s, t)=t^{m} t^{\operatorname{deg}(\gamma)} \gamma\left(\frac{s}{t}\right)$ and $\Omega(s, t)=t^{m^{\prime}} t^{\operatorname{deg}(\omega)} \omega\left(\frac{s}{t}\right)$, then

$$
\Gamma(s, t) \mid \Omega(s, t) \text { if and only if }\left\{\begin{array}{l}
\gamma(s) \mid \omega(s), \\
m \leq m^{\prime} .
\end{array}\right.
$$

3. Statement of the problems. The first problem we deal with in this paper is the following one.

Problem 3.1 (low rank perturbation for regular matrix pencils). Given two regular matrix pencils $A(s), B(s) \in \mathbb{F}[s]^{n \times n}$ and a nonnegative integer $r$, find necessary and sufficient conditions for the existence of a matrix pencil $P(s) \in \mathbb{F}[s]^{n \times n}$ such that $\operatorname{rank}(P(s)) \leq r$ and $A(s)+P(s) \stackrel{\text { s.e. }}{\sim} B(s)$.

The next lemma shows that the pencil $A(s)$ can be substituted by any other pencil strictly equivalent to $A(s)$.

Lemma 3.2. Let $A(s), B(s), P(s) \in \mathbb{F}[s]^{n \times n}$ be matrix pencils. Let $Q, R \in \mathrm{Gl}_{n}(\mathbb{F})$ and $A^{\prime}(s)=Q A(s) R$. If $A(s)+P(s) \stackrel{\text { s.e. }}{\sim} B(s)$, then $A^{\prime}(s)+Q P(s) R \stackrel{\text { s.e. }}{\sim} B(s)$.

Proof. If $B(s) \stackrel{\text { s.e. }}{\sim} A(s)+P(s)$, then $B(s) \stackrel{\text { s.e. }}{\sim} Q(A(s)+P(s)) R=A^{\prime}(s)+Q P(s) R$.

In the next lemma we see that the roles of the pencils $A(s)$ and $B(s)$ can be interchanged.

LEMmA 3.3. Let $A(s), B(s), P(s) \in \mathbb{F}[s]^{n \times n}$ be matrix pencils such that $A(s)+$ $P(s) \stackrel{\text { s.e. }}{\sim} B(s)$. Then there exists a matrix pencil $P^{\prime}(s) \in \mathbb{F}[s]^{n \times n}$ such that $B(s)+$ $P^{\prime}(s) \stackrel{\sim}{\text { s.e. }} A(s)$ and $\operatorname{rank}\left(P^{\prime}(s)\right)=\operatorname{rank}(P(s))$.

Proof. There exist $Q, R \in \mathrm{Gl}_{n}(\mathbb{F})$ such that $Q(A(s)+P(s)) R=B(s)$. Hence, $B(s)-Q P(s) R=Q A(s) R \stackrel{\text { s.e. }}{\sim} A(s)$ and the lemma follows with $P^{\prime}(s)=-Q P(s) R$. 
The solution to Problem 3.1 will allow us to also solve the following one.

Problem 3.4 (eigenvalue placement for regular matrix pencils under low rank perturbations). Given a regular matrix pencil $A(s) \in \mathbb{F}[s]^{n \times n}$, a nonnegative integer $r$ and a monic polynomial $0 \neq p(s) \in \mathbb{F}[s]$ with $\operatorname{deg}(p(s)) \leq n$, find necessary and sufficient conditions for the existence of a matrix pencil $P(s) \in \mathbb{F}[s]^{n \times n}$ such that $\operatorname{rank}(P(s)) \leq r$ and $\operatorname{det}(A(s)+P(s))=k p(s)$, with $k \in \mathbb{F}$.

Notice that in this problem we prescribe the spectrum $\Lambda(A(s)+P(s))$ and the algebraic multiplicities of every $\lambda \in \Lambda(A(s)+P(s))$. For $r=1$ and $\mathbb{F}=\mathbb{C}$ or $\mathbb{F}=\mathbb{R}$, a solution to Problem 3.4 is given in [9].

4. Low rank perturbation for regular matrix pencils. In this section we give a solution to Problem 3.1.

4.1. Necessary conditions. The next lemma was obtained in [13]. The result can also be found in [19, Theorem 1].

Lemma 4.1 (see $\left[13\right.$, Theorem 6.1]). Let $G(s), H(s) \in \mathbb{F}[s]^{m \times n}, n \leq m$, be polynomial matrices with invariant factors $\gamma_{1}(s)|\cdots| \gamma_{n}(s)$ and $\delta_{1}(s)|\cdots| \delta_{n}(s)$, respectively. Let $r$ be a nonnegative integer. There exist matrices $\hat{G}(s), \hat{H}(s) \in \mathbb{F}[s]^{m \times n}$ equivalent to $G(s)$ and $H(s)$, respectively, such that

$$
\operatorname{rank}(\hat{H}(s)-\hat{G}(s)) \leq r
$$

if and only if

$$
\delta_{i}(s)\left|\gamma_{i+r}(s), \quad \gamma_{i}(s)\right| \delta_{i+r}(s), \quad 1 \leq i \leq n-r .
$$

Remark 4.2. Condition (4.1) is equivalent to

$$
\gamma_{i-r}(s)\left|\delta_{i}(s)\right| \gamma_{i+r}(s), \quad 1 \leq i \leq n,
$$

and to

$$
\delta_{i-r}(s)\left|\gamma_{i}(s)\right| \delta_{i+r}(s), \quad 1 \leq i \leq n .
$$

Taking advantage of the above result, we obtain desired necessary conditions for solving Problem 3.1 in the next proposition.

Proposition 4.3. Let $A(s)=A_{0}+s A_{1}, B(s)=B_{0}+s B_{1} \in \mathbb{F}[s]^{n \times n}$ be regular matrix pencils. Let $P(s)=P_{0}+s P_{1} \in \mathbb{F}[s]^{n \times n}$ be a matrix pencil with $\operatorname{rank}(P(s))=r$ such that $A(s)+P(s) \stackrel{\text { s.e. }}{\sim} B(s)$. Let $\phi_{1}(s, t)|\cdots| \phi_{n}(s, t)$ and $\psi_{1}(s, t)|\cdots| \psi_{n}(s, t)$ be the homogeneous invariant factors of $A(s)$ and $B(s)$, respectively. Then

$$
\phi_{i-r}(s, t)\left|\psi_{i}(s, t)\right| \phi_{i+r}(s, t), \quad 1 \leq i \leq n .
$$

Proof. Let $\bar{A}(t)=t A_{0}+A_{1}, \bar{B}(t)=t B_{0}+B_{1}$, and $\bar{P}(t)=t P_{0}+P_{1}$.

Since $A(s)+P(s) \stackrel{\text { s.e. }}{\sim} B(s)$, we have that $A(s)+P(s) \sim B(s)$ and $\bar{A}(t)+\bar{P}(t) \sim$ $\bar{B}(t)$.

Let $\alpha_{1}(s)|\cdots| \alpha_{n}(s)$ and $\beta_{1}(s)|\cdots| \beta_{n}(s)$ be the invariant factors of $A(s)$ and $B(s)$, respectively. The invariant factors of $\bar{A}(t)=t A_{0}+A_{1}$ and $\bar{B}(t)=t B_{0}+B_{1}$ are, respectively,

$$
\begin{aligned}
& \bar{\alpha}_{i}(t)=k_{i} t^{m_{i}(\infty, A(s))} t^{\operatorname{deg}\left(\alpha_{i}\right)} \alpha_{i}\left(\frac{1}{t}\right), \quad 1 \leq i \leq n \quad\left(0 \neq k_{i} \in \mathbb{F}\right), \\
& \bar{\beta}_{i}(t)=k_{i}^{\prime} t^{m_{i}(\infty, B(s))} t^{\operatorname{deg}\left(\beta_{i}\right)} \beta_{i}\left(\frac{1}{t}\right), \quad 1 \leq i \leq n \quad\left(0 \neq k_{i}^{\prime} \in \mathbb{F}\right) .
\end{aligned}
$$


By Lemma 4.1,

$$
\alpha_{i-r}(s)\left|\beta_{i}(s)\right| \alpha_{i+r}(s), \quad 1 \leq i \leq n,
$$

and

$$
\bar{\alpha}_{i-r}(t)\left|\bar{\beta}_{i}(t)\right| \bar{\alpha}_{i+r}(t), \quad 1 \leq i \leq n .
$$

It follows from (4.4) that

$$
m_{i-r}(\infty, A(s)) \leq m_{i}(\infty, B(s)) \leq m_{i+r}(\infty, A(s)), \quad 1 \leq i \leq n .
$$

By Lemma 2.3, conditions (4.3) and (4.5) are equivalent to (4.2).

Remark 4.4. If $\operatorname{rank}(P(s))=r_{1} \leq r$, this proposition tells us that

$$
\phi_{i-r_{1}}(s, t)\left|\psi_{i}(s, t)\right| \phi_{i+r_{1}}(s, t), \quad 1 \leq i \leq n
$$

therefore,

$$
\phi_{i-r}(s, t)\left|\phi_{i-r_{1}}(s, t)\right| \psi_{i}(s, t)\left|\phi_{i+r_{1}}(s, t)\right| \phi_{i+r}(s, t), \quad 1 \leq i \leq n,
$$

hence, condition (4.2) is necessary for Problem 3.1.

Remark 4.5. Condition (4.2) is also equivalent to

$$
\psi_{i-r}(s, t)\left|\phi_{i}(s, t)\right| \psi_{i+r}(s, t), \quad 1 \leq i \leq n .
$$

Condition (4.2) can be stated in terms of the partial multiplicities of the elements of $\Lambda(A(s)) \cup \Lambda(B(s))$.

Corollary 4.6. Let $A(s), B(s) \in \mathbb{F}[s]^{n \times n}$ be regular matrix pencils. Let $P(s) \in$ $\mathbb{F}[s]^{n \times n}$ be a matrix pencil with $\operatorname{rank}(P(s))=r$ such that $A(s)+P(s) \stackrel{\text { s.e. }}{\sim} B(s)$. Then

$$
m_{i-r}(\lambda, A(s)) \leq m_{i}(\lambda, B(s)) \leq m_{i+r}(\lambda, A(s)), 1 \leq i \leq n, \quad \lambda \in \overline{\mathbb{F}} \cup\{\infty\} .
$$

Equivalently,

$$
m_{i-r}(\lambda, B(s)) \leq m_{i}(\lambda, A(s)) \leq m_{i+r}(\lambda, B(s)), 1 \leq i \leq n, \quad \lambda \in \overline{\mathbb{F}} \cup\{\infty\} .
$$

From Lemma 2.2 we conclude the following result (Corollary 4.7 for $r=1$ is proved in Proposition 4.2 of [9]).

Corollary 4.7. Let $A(s), B(s) \in \mathbb{F}[s]^{n \times n}$ be regular matrix pencils. Let $P(s) \in$ $\mathbb{F}[s]^{n \times n}$ be a matrix pencil with $\operatorname{rank}(P(s))=r$ such that $A(s)+P(s) \stackrel{\text { s.e. }}{\sim} B(s)$. Then

$$
w_{i}(\lambda, A(s))-r \leq w_{i}(\lambda, B(s)) \leq w_{i}(\lambda, A(s))+r, \quad 1 \leq i \leq n, \quad \lambda \in \overline{\mathbb{F}} \cup\{\infty\} .
$$

Equivalently,

$$
w_{i}(\lambda, B(s))-r \leq w_{i}(\lambda, A(s)) \leq w_{i}(\lambda, B(s))+r, \quad 1 \leq i \leq n, \quad \lambda \in \overline{\mathbb{F}} \cup\{\infty\} .
$$

Corollary 4.8. Let $A(s), B(s) \in \mathbb{F}[s]^{n \times n}$ be regular matrix pencils. Let $P(s) \in$ $\mathbb{F}[s]^{n \times n}$ be a matrix pencil with $\operatorname{rank}(P(s))=r$ such that $A(s)+P(s) \stackrel{\text { s.e. }}{\sim} B(s)$. Then

$$
\mu_{g}(\lambda, A(s))-r \leq \mu_{g}(\lambda, B(s)) \leq \mu_{g}(\lambda, A(s))+r, \quad \lambda \in \overline{\mathbb{F}} \cup\{\infty\} .
$$


4.2. Sufficiency of the conditions. For the case when $A(s)=s I_{n}-A, B(s)=$ $s I_{n}-B$, with $A, B \in \mathbb{F}^{n \times n}$, a solution to Problem 3.1 is given in [21] for $r=1$ and in [20] and [22] for the general case. Taking advantage of this result (see Proposition 4.9) we prove in Corollary 4.10 the sufficiency of conditions (4.2) for pencils without infinite elementary divisors.

Proposition 4.9 (see [20, Theorem 1], [22, Theorem 3]). Let $G \in \mathbb{F}^{n \times n}$ and $\gamma_{1}(s)|\cdots| \gamma_{n}(s)$ be its invariant factors. Let $\delta_{1}(s)|\cdots| \delta_{n}(s)$ be monic polynomials such that $\sum_{i=1}^{n} \operatorname{deg}\left(\delta_{i}(s)\right)=n$. Let $r$ be a nonnegative integer. Then there exists a matrix $P \in \mathbb{F}^{n \times n}$ such that $\operatorname{rank}(P) \leq r$ and $G+P$ has $\delta_{1}(s)|\cdots| \delta_{n}(s)$ as invariant factors if and only if

$$
\delta_{i-r}(s)\left|\gamma_{i}(s)\right| \delta_{i+r}(s), \quad 1 \leq i \leq n
$$

Corollary 4.10. Let $A(s)=A_{0}+s A_{1}, B(s)=B_{0}+s B_{1} \in \mathbb{F}[s]^{n \times n}$ be such that $\operatorname{det}\left(A_{1}\right) \neq 0$ and $\operatorname{det}\left(B_{1}\right) \neq 0$. Let $\phi_{1}(s, t)|\cdots| \phi_{n}(s, t)$ and $\psi_{1}(s, t)|\cdots|$ $\psi_{n}(s, t)$ be the homogeneous invariant factors of $A(s)$ and $B(s)$, respectively. Let $r$ be a nonnegative integer. If (4.2) is satisfied, then there exists $P_{0} \in \mathbb{F}^{n \times n}$ such that $\operatorname{rank}\left(P_{0}\right) \leq r$ and $A(s)+P_{0} \stackrel{\text { s.e. }}{\sim} B(s)$.

Proof. Recall that two square matrices $G, H \in \mathbb{F}^{n \times n}$ are similar if and only if $\left(s I_{n}-G\right) \sim\left(s I_{n}-H\right)$ and that the invariant factors of $G \in \mathbb{F}^{n \times n}$ are those of $s I_{n}-G$.

Let $\alpha_{1}(s)|\cdots| \alpha_{n}(s)$ and $\beta_{1}(s),|\cdots| \beta_{n}(s)$ be the invariant factors of $A(s)$ and $B(s)$, respectively. Since $\operatorname{det}\left(A_{1}\right) \neq 0$ and $\operatorname{det}\left(B_{1}\right) \neq 0$, these pencils do not have infinite elementary divisors; therefore,

$$
m_{1}(\infty, A(s))=\cdots=m_{n}(\infty, A(s))=0, \quad m_{1}(\infty, B(s))=\cdots=m_{n}(\infty, B(s))=0 .
$$

As a consequence, (4.2) is equivalent to

$$
\alpha_{i-r}(s)\left|\beta_{i}(s)\right| \alpha_{i+r}(s), \quad 1 \leq i \leq n .
$$

We have that $A(s) \stackrel{\text { s.e. }}{\sim} A_{1}^{-1}\left(A_{0}+A_{1} s\right)=A_{1}^{-1} A_{0}+s I_{n}$ and $B(s) \stackrel{\text { s.e. }}{\sim} B_{1}^{-1}\left(B_{0}+\right.$ $\left.B_{1} s\right)=B_{1}^{-1} B_{0}+s I_{n}$, hence the invariant factors of $-A_{1}^{-1} A_{0}$ and $-B_{1}^{-1} B_{0}$ are $\alpha_{1}(s)$ $\cdots \mid \alpha_{n}(s)$ and $\beta_{1}(s),|\cdots| \beta_{n}(s)$, respectively. By Proposition 4.9, there exists $P \in \mathbb{F}^{n \times n}$ such that $\operatorname{rank}(P) \leq r$ and $P+A_{1}^{-1} A_{0}+s I_{n} \sim B_{1}^{-1} B_{0}+s I_{n}$, hence $P+A_{1}^{-1} A_{0}+s I_{n} \stackrel{\text { s.e. }}{\sim} B(s)$. Setting $P_{0}=A_{1} P$, we have that $\operatorname{rank}\left(P_{0}\right) \leq r$ and by Lemma 3.2, $A(s)+P_{0} \stackrel{\text { s.e. }}{\sim} B(s)$.

As an immediate consequence of Corollary 4.10, we obtain in Corollary 4.11 the sufficiency of conditions (4.2) for pencils not having the eigenvalue zero.

Corollary 4.11. Let $A(s)=A_{0}+s A_{1}, B(s)=B_{0}+s B_{1} \in \mathbb{F}[s]^{n \times n}$ be such that $\operatorname{det}\left(A_{0}\right) \neq 0$ and $\operatorname{det}\left(B_{0}\right) \neq 0$. Let $\phi_{1}(s, t)|\cdots| \phi_{n}(s, t)$ and $\psi_{1}(s, t)|\cdots|$ $\psi_{n}(s, t)$ be the homogeneous invariant factors of $A(s)$ and $B(s)$, respectively. Let $r$ be a nonnegative integer. If (4.2) is satisfied, then there exists $P_{1} \in \mathbb{F}^{n \times n}$ such that $\operatorname{rank}\left(P_{1}\right) \leq r$ and $A(s)+s P_{1} \stackrel{\text { s.e. }}{\sim} B(s)$.

Proof. The homogeneous invariant factors of the pencils $\bar{A}(t)=t A_{0}+A_{1}$ and $\bar{B}(t)=t B_{0}+B_{1}$ are $k_{1} \phi_{1}(s, t), \ldots, k_{n} \phi_{n}(s, t)$ and $k_{1}^{\prime} \psi_{1}(s, t), \ldots, k_{n}^{\prime} \psi_{n}(s, t)$, respectively, for some nonzero scalars $k_{1}, \ldots, k_{n}, k_{1}^{\prime}, \ldots, k_{n}^{\prime} \in \mathbb{F}$.

By Corollary 4.10, there exists $P_{1} \in \mathbb{F}^{n \times n}$ such that $\operatorname{rank}\left(P_{1}\right) \leq r$ and $\bar{A}(t)+P_{1}=$ $A_{1}+P_{1}+t A_{0} \stackrel{\text { s.e. }}{\sim} \bar{B}(t)=B_{1}+t B_{0}$. Therefore, $A(s)+s P_{1}=s\left(A_{1}+P_{1}\right)+A_{0} \stackrel{\text { s.e. }}{\sim}$ $s B_{1}+B_{0}=B(s)$. 
The restriction on the field $\mathbb{F}$ introduced in the next corollary allows us to perform a change of variable over an arbitrary regular pencil, which turns it into a new pencil without the zero eigenvalue.

Corollary 4.12. Let $A(s)=A_{0}+s A_{1}, B(s)=B_{0}+s B_{1} \in \mathbb{F}[s]^{n \times n}$ be regular pencils, and assume that there exists $c \in \mathbb{F}$ such that $c \notin \Lambda_{f}(A(s)) \cup \Lambda_{f}(B(s))$. Let $\phi_{1}(s, t)|\cdots| \phi_{n}(s, t)$ and $\psi_{1}(s, t)|\cdots| \psi_{n}(s, t)$ be the homogeneous invariant factors of $A(s)$ and $B(s)$, respectively. Let $r$ be a nonnegative integer. If (4.2) is satisfied, then there exists $P \in \mathbb{F}^{n \times n}$ such that $\operatorname{rank}(P) \leq r$ and $A(s)+(s-c) P \stackrel{\text { s.e. }}{\sim} B(s)$.

Proof. As $c \notin \Lambda_{f}(A(s))$ and $c \notin \Lambda_{f}(B(s))$, we have that $\operatorname{det}\left(A_{0}+c A_{1}\right) \neq 0$ and $\operatorname{det}\left(B_{0}+c B_{1}\right) \neq 0$.

Let us consider the regular matrix pencils

$$
\begin{aligned}
& A^{\prime}(y):=\left(A_{0}+c A_{1}\right)+y A_{1}=A_{0}+(y+c) A_{1}=A(y+c) \in \mathbb{F}[y]^{n \times n}, \\
& B^{\prime}(y):=\left(B_{0}+c B_{1}\right)+y B_{1}=B_{0}+(y+c) B_{1}=B(y+c) \in \mathbb{F}[y]^{n \times n} .
\end{aligned}
$$

The associated homogeneous pencils are

$$
\begin{aligned}
& A^{\prime}(y, z)=z\left(A_{0}+c A_{1}\right)+y A_{1}=z A_{0}+(c z+y) A_{1} \in \mathbb{F}[z, y]^{n \times n}, \\
& B^{\prime}(y, z)=z\left(B_{0}+c B_{1}\right)+y B_{1}=z B_{0}+(c z+y) B_{1} \in \mathbb{F}[z, y]^{n \times n} .
\end{aligned}
$$

Therefore, the homogeneous invariant factors of $A^{\prime}(y)$ and $B^{\prime}(y)$ are

$$
\phi_{i}^{\prime}(y, z)=\phi_{i}(c z+y, z), \quad 1 \leq i \leq n,
$$

and

$$
\psi_{i}^{\prime}(y, z)=\psi_{i}(c z+y, z), \quad 1 \leq i \leq n,
$$

respectively. Then, condition (4.2) implies

$$
\phi_{i-r}^{\prime}(y, z)\left|\psi_{i}^{\prime}(y, z)\right| \phi_{i+r}^{\prime}(y, z), \quad 1 \leq i \leq n .
$$

By Corollary 4.11, there exists $P \in \mathbb{F}^{n \times n}$ such that $\operatorname{rank}(P) \leq r$ and $A^{\prime}(y)+y P \stackrel{\text { s.e. }}{\sim}$ $B^{\prime}(y)$. Then $A(s)+(s-c) P=A^{\prime}(s-c)+(s-c) P \stackrel{\text { s.e. }}{\sim} B^{\prime}(s-c)=B(s)$.

The next theorem gives a complete solution to Problem 3.1 under a restriction on the field $\mathbb{F}$.

TheOREM 4.13. Let $A(s), B(s) \in \mathbb{F}[s]^{n \times n}$ be regular matrix pencils. Let $\phi_{1}(s, t) \mid$ $\ldots \mid \phi_{n}(s, t)$ and $\psi_{1}(s, t)|\cdots| \psi_{n}(s, t)$ be the homogeneous invariant factors of $A(s)$ and $B(s)$, respectively, and assume that $\mathbb{F} \cup\{\infty\} \nsubseteq \Lambda(A(s)) \cup \Lambda(B(s))$. Let $r$ be a nonnegative integer. There exists a matrix pencil $P(s) \in \mathbb{F}[s]^{n \times n}$ such that $\operatorname{rank}(P(s)) \leq r$ and $A(s)+P(s) \stackrel{\text { s.e. }}{\sim} B(s)$ if and only if (4.2) holds.

Proof. The necessity follows from Proposition 4.3 and Remark 4.4.

Assume that (4.2) holds. As $\mathbb{F} \cup\{\infty\} \nsubseteq \Lambda(A(s)) \cup \Lambda(B(s))$, there exists $c \in \mathbb{F} \cup\{\infty\}$ such that $c \notin \Lambda(A(s)) \cup \Lambda(B(s))$. If $c=\infty$, then we apply Corollary 4.10, and if $c \neq \infty$, then we apply Corollary 4.12 .

In Corollary 4.14 we restate Theorem 4.13 in terms of the partial multiplicities of the elements of $\Lambda(A(s)) \cup \Lambda(B(s))$.

Corollary 4.14. Let $A(s), B(s) \in \mathbb{F}[s]^{n \times n}$ be regular matrix pencils. Assume that $\mathbb{F} \cup\{\infty\} \nsubseteq \Lambda(A(s)) \cup \Lambda(B(s))$. Let $r$ be a nonnegative integer. There exists a matrix pencil $P(s) \in \mathbb{F}[s]^{n \times n}$ such that $\operatorname{rank}(P(s)) \leq r$ and $A(s)+P(s) \stackrel{\text { s.e. }}{\sim} B(s)$ if and only if (4.6) (equivalently, (4.7)) holds. 
Remark 4.15. In Theorem 4.13, the condition on the field $\mathbb{F}$ means that there exists an element $c \in \mathbb{F} \cup\{\infty\}$ which is neither an eigenvalue of $A(s)$ nor of $B(s)$. Observe that if $\# \mathbb{F}>2 n$, this condition is automatically satisfied. In the case that $\# \mathbb{F} \leq 2 n$, Theorem 4.13 can still be applied, as we show in Example 4.16. Moreover, the condition $\mathbb{F} \cup\{\infty\} \nsubseteq \Lambda(A(s)) \cup \Lambda(B(s))$ is not always necessary. In Theorem 4.17 below we prove that even in the case that $\mathbb{F} \cup\{\infty\} \subseteq \Lambda(A(s)) \cup \Lambda(B(s)$ ), for certain special structures of $A(s)$ and $B(s)$, condition (4.2) is sufficient. We illustrate the case in Example 4.18.

Example 4.16. This example is valid for any field $\mathbb{F}$, including $\mathbb{F}=\mathbb{Z}_{2}$.

Let

$$
A(s)=\left[\begin{array}{lll}
s & 0 & 0 \\
0 & s & 1 \\
0 & 0 & s
\end{array}\right], \quad B(s)=\left[\begin{array}{lll}
1 & s & 0 \\
0 & 1 & 0 \\
0 & 0 & s
\end{array}\right] .
$$

The homogeneous invariant factors of $A(s)$ and $B(s)$ are $\phi_{1}(s, t)=1, \phi_{2}(s, t)=$ $s, \phi_{3}(s, t)=s^{2}$ and $\psi_{1}(s, t)=\psi_{2}(s, t)=1, \psi_{3}(s, t)=t^{2} s$, respectively. We have that $1 \notin\{0, \infty\}=\Lambda(A(s)) \cup \Lambda(B(s))$ and

$$
\phi_{i-1}(s, t)\left|\psi_{i}(s, t)\right| \phi_{i+1}(s, t), \quad i=1,2,3 .
$$

Therefore, there exists a matrix pencil $P(s) \in \mathbb{F}[s]^{3 \times 3}$ such that $\operatorname{rank} P(s) \leq 1$ and $A(s)+P(s) \stackrel{\text { s.e. }}{\sim} B(s)$. In fact,

$$
A(s)+\left[\begin{array}{ccc}
0 & 0 & 0 \\
0 & 0 & 0 \\
0 & 1 & -s
\end{array}\right]=\left[\begin{array}{ccc}
s & 0 & 0 \\
0 & s & 1 \\
0 & 1 & 0
\end{array}\right] \stackrel{\text { s.e. }}{\sim} B(s) .
$$

Theorem 4.17. Let $A(s), B(s) \in \mathbb{F}[s]^{n \times n}$ be regular matrix pencils. Let $\phi_{1}(s, t) \mid$ $\cdots \mid \phi_{n}(s, t)$ and $\psi_{1}(s, t)|\cdots| \psi_{n}(s, t)$ be the homogeneous invariant factors of $A(s)$ and $B(s)$, respectively, and assume that for some $\lambda_{0} \in \mathbb{F} \cup\{\infty\}$,

$$
m_{i}\left(\lambda_{0}, A(s)\right)=m_{i}\left(\lambda_{0}, B(s)\right), \quad 1 \leq i \leq n .
$$

Let $r$ be a nonnegative integer. There exists a matrix pencil $P(s) \in \mathbb{F}[s]^{n \times n}$ such that $\operatorname{rank}(P(s)) \leq r$ and $A(s)+P(s) \stackrel{\text { s.e. }}{\sim} B(s)$ if and only if (4.2) holds.

Proof. If

$$
m_{i}\left(\lambda_{0}, A(s)\right)=m_{i}\left(\lambda_{0}, B(s)\right)=0, \quad 1 \leq i \leq n,
$$

then $\lambda_{0} \notin \Lambda(A(s)) \cup \Lambda(B(s))$ and we can apply Theorem 4.13. In fact, condition (4.9) implies the condition $\mathbb{F} \cup\{\infty\} \not \subseteq \Lambda(A(s)) \cup \Lambda(B(s))$ of Theorem 4.13.

In any other case, let $\mu_{a}\left(\lambda_{0}, A(s)\right)=\mu_{a}\left(\lambda_{0}, B(s)\right):=n_{1}$. Then

$$
A(s) \stackrel{\text { s.e. }}{\sim} A^{\prime}(s)=\left[\begin{array}{cc}
A_{11}(s) & 0 \\
0 & A_{22}(s)
\end{array}\right], \quad B(s) \stackrel{\text { s.e. }}{\sim} B^{\prime}(s)=\left[\begin{array}{cc}
A_{11}(s) & 0 \\
0 & B_{22}(s)
\end{array}\right],
$$

where $A_{11}(s) \in \mathbb{F}[s]^{n_{1} \times n_{1}}, A_{22}(s), B_{22}(s) \in \mathbb{F}[s]^{\left(n-n_{1}\right) \times\left(n-n_{1}\right)}$ are regular pencils such that $\Lambda\left(A_{11}(s)\right)=\left\{\lambda_{0}\right\}, m_{i}\left(\lambda_{0}, A_{11}(s)\right)=m_{n-n_{1}+i}\left(\lambda_{0}, A(s)\right)=m_{n-n_{1}+i}\left(\lambda_{0}, B(s)\right)$ for $1 \leq i \leq n_{1}$ and the homogeneous invariant factors of $A_{22}(s)$ and $B_{22}(s)$ are $\phi_{i}^{\prime}(s, t)=$ $\phi_{n_{1}+i}(s, t) /\left(s-\lambda_{0} t\right)^{n_{1}+i}$ and $\psi_{i}^{\prime}(s, t)=\psi_{n_{1}+i}(s, t) /\left(s-\lambda_{0} t\right)^{n_{1}+i}, 1 \leq i \leq n-n_{1}$, respectively. From (4.2),

$$
\phi_{i-r}^{\prime}(s, t)\left|\psi_{i}^{\prime}(s, t)\right| \phi_{i+r}^{\prime}(s, t), \quad 1 \leq i \leq n-n_{1} .
$$


By Theorem 4.13, there exists a matrix pencil $P_{22}(s) \in \mathbb{F}[s]^{\left(n-n_{1}\right) \times\left(n-n_{1}\right)}$ such that $\operatorname{rank} P_{22}(s) \leq r$ and $A_{22}(s)+P_{22}(s) \stackrel{\text { s.e. }}{\sim} B_{22}(s)$. Taking

$$
P(s)=\left[\begin{array}{cc}
0 & 0 \\
0 & P_{22}(s)
\end{array}\right] \in \mathbb{F}[s]^{n \times n},
$$

we have that $A^{\prime}(s)+P(s) \stackrel{\text { s.e. }}{\sim} B(s)$ and $\operatorname{rank} P(s) \leq r$.

Example 4.18. Assume that $\mathbb{F}=\mathbb{Z}_{2}, n=5$, and the homogeneous invariant factors of $A(s)$ and $B(s)$ are $\phi_{1}(s, t)=\phi_{2}(s, t)=\phi_{3}(s, t)=1, \phi_{4}(s, t)=s(s-$ $t), \phi_{5}(s, t)=s^{2}(s-t)$ and $\psi_{1}(s, t)=\psi_{2}(s, t)=\psi_{3}(s, t)=1, \psi_{4}(s, t)=(s-t), \psi_{5}(s, t)=$ $t^{2} s(s-t)$, respectively. Then

$$
\Lambda(A(s))=\{0,1\}, \quad \Lambda(B(s))=\{0,1, \infty\},
$$

and $\mathbb{F} \cup\{\infty\}=\Lambda(A(s)) \cup \Lambda(B(s))=\{0,1, \infty\}$. But

$$
\left(m_{1}(1, A(s)), \ldots, m_{5}(1, A(s))\right)=\left(m_{1}(1, B(s)), \ldots, m_{5}(1, B(s))\right)=(0,0,0,1,1) .
$$

Then,

$$
A(s) \stackrel{\text { s.e. }}{\sim} A^{\prime}(s)=\left[\begin{array}{cc}
A_{11}(s) & 0 \\
0 & A_{22}(s)
\end{array}\right], \quad B(s) \stackrel{\text { s.e. }}{\sim} B^{\prime}(s)=\left[\begin{array}{cc}
A_{11}(s) & 0 \\
0 & B_{22}(s)
\end{array}\right],
$$

where

$$
A_{11}(s)=\left[\begin{array}{cc}
s-1 & 0 \\
0 & s-1
\end{array}\right] .
$$

$A_{22}(s), B_{22}(s) \in \mathbb{F}[s]^{3 \times 3}$, and the homogeneous invariant factors of $A_{22}(s)$ and $B_{22}(s)$ are $\phi_{1}^{\prime}(s, t)=1, \phi_{2}^{\prime}(s, t)=s, \phi_{3}^{\prime}(s, t)=s^{2}$ and $\psi_{1}^{\prime}(s, t)=\psi_{2}^{\prime}(s, t)=1, \psi_{3}^{\prime}(s, t)=t^{2} s$, respectively. Hence (see Example 4.16), there exists a matrix pencil $P_{22}(s) \in \mathbb{F}[s]^{3 \times 3}$ such that $\operatorname{rank} P_{22}(s) \leq 1$ and $A_{22}(s)+P_{22}(s) \stackrel{\text { s.e. }}{\sim} B_{22}(s)$. Taking

$$
P(s)=\left[\begin{array}{cc}
0 & 0 \\
0 & P_{22}(s)
\end{array}\right] \in \mathbb{F}[s]^{(2+3) \times(2+3)},
$$

we have that $A^{\prime}(s)+P(s) \stackrel{\text { s.e. }}{\sim} B(s)$ and $\operatorname{rank} P(s) \leq 1$.

5. Eigenvalue placement for regular matrix pencils under low rank perturbations. In this section we solve Problem 3.4.

For $\lambda \in \overline{\mathbb{F}} \cup\{\infty\}$, we will denote $M_{r}(\lambda, A(s)):=\sum_{i=n-r+1}^{n} m_{i}(\lambda, A(s))$ and $M_{r}(A(s)):=\sum_{\lambda \in \Lambda(A(s))} M_{r}(\lambda, A(s))$.

Proposition 5.1. Let $A(s), P(s) \in \mathbb{F}[s]^{n \times n}$ be matrix pencils with $\operatorname{rank}(P(s))=$ $r$. Assume that $A(s)$ and $A(s)+P(s)$ are regular. Let $\alpha_{1}(s)|\cdots| \alpha_{n}(s)$ be the invariant factors of $A(s)$ and $p(s)=\operatorname{det}(A(s)+P(s))$. Then

$$
\begin{aligned}
& \alpha_{1}(s) \ldots \alpha_{n-r}(s) \mid p(s) \\
& \mu_{a}(\infty, A(s))-M_{r}(\infty, A(s)) \leq n-\operatorname{deg}(p(s)) .
\end{aligned}
$$

Proof. Let $B(s)=A(s)+P(s)$, and let $\beta_{1}(s)|\cdots| \beta_{n}(s)$ be its invariant factors. By Proposition 4.3 and Lemma 2.3, condition (4.8) and

$$
m_{i-r}(\infty, A(s)) \leq m_{i}(\infty, B(s)) \leq m_{i+r}(\infty, A(s)), \quad 1 \leq i \leq n,
$$


hold. As $p(s)=k \beta_{1}(s) \ldots \beta_{n}(s)$, with $0 \neq k \in \mathbb{F}$, we have that

$$
\begin{aligned}
& \alpha_{1}(s) \ldots \alpha_{n-r}(s) \mid p(s), \\
& \sum_{i=1}^{n} m_{i-r}(\infty, A(s)) \leq \sum_{i=1}^{n} m_{i}(\infty, B(s)) .
\end{aligned}
$$

But $\sum_{i=1}^{n} m_{i-r}(\infty, A(s))=\mu_{a}(\infty, A(s))-M_{r}(\infty, A(s))$ and $\sum_{i=1}^{n} m_{i}(\infty, B(s))=$ $\mu_{a}(\infty, B(s))=n-\operatorname{deg}(p(s))$.

From Proposition 5.1 we derive in Corollary 5.2 a lower bound for the algebraic multiplicity of the eigenvalues of the perturbed pencil.

Corollary 5.2. Let $A(s), P(s) \in \mathbb{F}[s]^{n \times n}$ be matrix pencils with $\operatorname{rank}(P(s))=r$. Assume that $A(s)$ and $A(s)+P(s)$ are regular. Then

$$
\mu_{a}(\lambda, A(s))-M_{r}(\lambda, A(s)) \leq \mu_{a}(\lambda, A(s)+P(s)), \quad \lambda \in \overline{\mathbb{F}} \cup\{\infty\} .
$$

Remark 5.3. Under the conditions of Corollary 5.2, if for $\lambda \in \overline{\mathbb{F}} \cup\{\infty\}$ we take $d(\lambda)=\mu_{a}(\lambda, A(s)+P(s))-\mu_{a}(\lambda, A(s))+M_{r}(\lambda, A(s))$ and denote $\mathcal{F}=\Lambda(A(s)) \cup$ $\Lambda(A(s)+P(s)) \cup\{\infty\}$, then

$$
\sum_{\lambda \in \mathcal{F}} d(\lambda)=n-n+\sum_{\lambda \in \Lambda(A(s))} M_{r}(\lambda, A(s))=M_{r}(A(s)) .
$$

Condition (5.3) implies $d(\lambda) \geq 0$ for all $\lambda \in \overline{\mathbb{F}} \cup\{\infty\}$. Hence,

$$
d(\lambda) \leq M_{r}(A(s)), \quad \lambda \in \overline{\mathbb{F}} \cup\{\infty\} .
$$

Therefore, (5.3) implies

$$
\mu_{a}(\lambda, A(s)+P(s)) \leq \mu_{a}(\lambda, A(s))-M_{r}(\lambda, A(s))+M_{r}(A(s)), \quad \lambda \in \overline{\mathbb{F}} \cup\{\infty\} .
$$

We notice that conditions (5.3) and (5.4) for $r=1$ are those of [9, Theorem 4.4].

The next theorem gives a solution to Problem 3.4 under a restriction on the field $\mathbb{F}$. We will use the following notation for a given $p(s) \in \mathbb{F}[s]$ with $\operatorname{deg}(p(s)) \leq n$ :

$$
\begin{gathered}
\Lambda(p(s)):=\{\lambda \in \overline{\mathbb{F}}: p(\lambda)=0\} \text { if } \operatorname{deg}(p(s))=n, \\
\Lambda(p(s)):=\{\lambda \in \overline{\mathbb{F}}: p(\lambda)=0\} \cup\{\infty\} \text { if } \operatorname{deg}(p(s))<n .
\end{gathered}
$$

TheOREM 5.4. Let $A(s) \in \mathbb{F}[s]^{n \times n}$ be a regular matrix pencil and $\alpha_{1}(s)|\cdots|$ $\alpha_{n}(s)$ be its invariant factors. Let $p(s) \in \mathbb{F}[s]$ be a nonzero monic polynomial with $\operatorname{deg}(p(s)) \leq n$. Assume that $\mathbb{F} \cup\{\infty\} \nsubseteq \Lambda(A(s)) \cup \Lambda(p(s))$. Let $r$ be a nonnegative integer. There exists a matrix pencil $P(s) \in \mathbb{F}[s]^{n \times n}$ such that $\operatorname{rank}(P(s)) \leq r$ and $\operatorname{det}(A(s)+P(s))=k p(s)$ with $0 \neq k \in \mathbb{F}$ if and only if (5.1) holds.

Proof. The necessity follows from Proposition 5.1.

Assume now that (5.1) holds. Then there exists $\gamma(s) \in \mathbb{F}[s]$ such that

$$
p(s)=\alpha_{1}(s) \ldots \alpha_{n-r}(s) \gamma(s)
$$

and

$$
d=n-\operatorname{deg}(p(s))-\mu_{a}(\infty, A(s))+M_{r}(\infty, A(s)) \geq 0 .
$$

Let us define

$$
\beta_{i}(s):=\alpha_{i-r}(s), 1 \leq i \leq n-1, \quad \beta_{n}(s):=\alpha_{n-r}(s) \gamma(s),
$$




$$
m_{i}^{\prime}:=m_{i-r}(\infty, A(s)), 1 \leq i \leq n-1, \quad m_{n}^{\prime}:=m_{n-r}(\infty, A(s))+d .
$$

Then

$$
\begin{gathered}
\beta_{1}(s)|\cdots| \beta_{n}(s), \quad m_{1}^{\prime} \leq \cdots \leq m_{n}^{\prime}, \\
\sum_{i=1}^{n} \operatorname{deg}\left(\beta_{i}(s)\right)+\sum_{i=1}^{n} m_{i}^{\prime}=\operatorname{deg}(p(s))+\mu_{a}(\infty, A(s))-M_{r}(\infty, A(s))+d=n .
\end{gathered}
$$

Let $B(s)$ be a pencil with invariant factors $\beta_{1}(s)|\cdots| \beta_{n}(s)$ and $m_{i}(\infty, B(s))=$ $m_{i}^{\prime}, 1 \leq i \leq n$. Then, $B(s)$ is regular and satisfies (4.8) and (5.2).

By Theorem 4.13, there exists a pencil $P(s) \in \mathbb{F}[s]^{n \times n}$ such that $\operatorname{rank}(P(s)) \leq r$ and $A(s)+P(s) \stackrel{\text { s.e. }}{\sim} B(s)$. Then there exist $0 \neq k_{1}, k_{2} \in \mathbb{F}$ such that

$$
\begin{gathered}
\operatorname{det}(A(s)+P(s))=k_{1} \operatorname{det}(B(s))=k_{1} k_{2} \beta_{1}(s) \ldots \beta_{n}(s)=k_{1} k_{2} \alpha_{1}(s) \ldots \alpha_{n-r}(s) \gamma(s) \\
=k_{1} k_{2} p(s), \quad 0 \neq k_{1} k_{2} \in \mathbb{F} .
\end{gathered}
$$

6. Conclusions. Given a regular matrix pencil, we have completely characterized the Weierstrass structure of a regular pencil obtained by a low rank perturbation of it. The conditions obtained are expressed both in terms of the homogeneous invariant factors and in terms of the partial multiplicities of the eigenvalues. The necessity of the conditions holds over arbitrary fields and the sufficiency over fields with a sufficient number of elements.

We also determine the possible eigenvalues and their algebraic multiplicities of a regular matrix pencil obtained by a low rank perturbation of another regular one. An open problem related to this study is to develop efficient methods for constructing the pencils $P(s)$ whose existence is established in Theorem 5.4.

For singular matrix pencils, the general case remains open. As mentioned in the introduction, for a particular kind of pencil the problem is solved in [6] and the generic behavior is described in [3].

Acknowledgment. We would like to thank the reviewers for their remarks and comments, which have contributed to improving the presentation of this paper.

\section{REFERENCES}

[1] L. BATZKe, Generic rank-one perturbations of structured regular matrix pencils, Linear Algebra Appl., 458 (2014), pp. 638-670.

[2] L. BATZKE, Sign characteristics of regular Hermitian matrix pencils under generic rank-1 and rank-2 perturbations, Electron. J. Linear Algebra, 30 (2015), pp. 760-794.

[3] F. De TERÁN AND F. Dopico, Low rank perturbation of Kronecker structures without full rank, SIAM J. Matrix Anal. Appl., 29 (2007), pp. 496-529, https://doi.org/10.1137/060659922.

[4] F. De Terán and F. Dopico, Generic change of the partial multiplicities of regular matrix pencils under low-rank perturbations, SIAM J. Matrix Anal. Appl., 37 (2016), pp. 823-835, https://doi.org/10.1137/15M1022069.

[5] F. De Terán, F. Dopico, and J. Moro, Low rank perturbation of Weierstrass structure, SIAM J. Matrix Anal. Appl., 30 (2008), pp. 538-547, https://doi.org/10.1137/050633020.

[6] M. Dodig And M. Stošić, The rank distance problem for pairs of matrices and a completion of quasi-regular matrix pencils, Linear Algebra Appl., 457 (2014), pp. 313-347.

[7] S. Friedland, Matrices-Algebra, Analysis and Applications, World Scientific, Hackensack, NJ, 2016.

[8] F. Gantmacher, Matrix Theory, Vols. I, II, Chelsea, New York, 1974.

[9] H. Gernandt AND C. TRunk, Eigenvalue placement for regular matrix pencils with rank one perturbations, SIAM J. Matrix Anal. Appl., 38 (2017), pp. 134-154, https://doi.org/10. $1137 / 16 \mathrm{M} 1066877$. 
[10] M. K. I. Gohberg And F. van Schagen, Partially Specified Matrices and Operators: Classification, Completion, Applications, Birkhäuser Verlag, Basel, 1995.

[11] L. Leiben, F. Martínez Pería, F. Philipp, C. Trunk, H. Winkler, Finite Rank Perturbations of Linear Relations and Singular Matrix Pencils, preprint, https://arxiv.org/abs/ 1806.07513, 2018.

[12] R. Lippert and G. Strang, The Jordan forms of $A B$ and $B A$, Electron. J. Linear Algebra, 18 (2009), pp. 281-288.

[13] E. MARques DE SÀ, Imersão de matrices e entrelaçamento de factores invariantes, Ph.D. thesis, University of Coimbra, Coimbra, Portugal, 1979.

[14] C. Mehl, V. Mehrmann, A. Ran, and L. Rodman, Eigenvalue perturbation theory of classes of structured matrices under generic structured rank one perturbations, Linear Algebra Appl., 435 (2011), pp. 687-716.

[15] J. Moro And F. Dopico, Low rank perturbation of Jordan structure, SIAM J. Matrix Anal. Appl., 25 (2003), pp. 495-506, https://doi.org/10.1137/S0895479802417118.

[16] A. RocA, Asignación de Invariantes en Sistemas de Control, Ph.D. thesis, Universitat Politècnica València, València, Spain, 2003.

[17] S. V. Savchenko, Typical changes in spectral properties under perturbations by a rank-one operator, Math. Notes, 74 (2003), pp. 557-568.

[18] S. V. SAVChENKo, On the change in the spectral properties of a matrix under perturbations of sufficiently low rank, Funct. Anal. Appl., 38 (2004), pp. 69-71.

[19] F. Silva, The rank of the difference of matrices with prescribed invariant factors, Linear and Multilinear Algebra, 24 (1988), pp. 59-63.

[20] F. Silva, The rank of the difference of matrices with prescribed similarity classes, Linear and Multilinear Algebra, 24 (1988), pp. 51-58.

[21] R. Thompson, Invariant factors under rank one perturbations, Canad. J. Math., 32 (1980), pp. 240-245.

[22] I. Zaballa, Pole assignment and additive perturbations of fixed rank, SIAM J. Matrix Anal. Appl., 12 (1991), pp. 16-23, https://doi.org/10.1137/0612003. 